Background Free fetal DNA (ffDNA) testing for aneuploidy has recently started being offered to all women in our institution who are high risk for trisomy 21 (aged 35 or over at estimated date of delivery). We therefore examined the uptake rate of ffDNA for aneuploidy screening in women of advanced maternal age who had been seen by a genetic counsellor.

As this test has much higher sensitivity than other screening tests, we hypothesised that more women will have ffDNA than other forms of screening.

Methods We included 258 women who had singleton pregnancies and who were of advanced maternal age. We undertook a retrospective analysis of electronic chart data. Chi squared statistical analysis was performed on the data to determine statistical significance between groups based on age, nuchal thickness and parity.

Results 118 (46\%) women had ffDNA for screening for aneuploidy, the remaining 140 (54\%) chose either other screening options or no screening. Statistically there was no difference in choice between groups of women dependent on their calculated risk based on their age $(p=0.15)$. The size of the nuchal lucency measurement (within normal range) had no statistical effect on the choice $(p=0.16)$. Women who were primiparous were no more likely to have ffDNA than multiparous women $(p=0.4)$.

Conclusions Although ffDNA is a more accurate way of screening for aneuploidy, the majority of women are still using other forms of screening. We found that the uptake of ffDNA testing was not statistically significantly affected by age, nuchal thickness or parity.

\section{PF.39 CLINICAL RELEVANCE OF FETAL WEIGHT ESTIMATION IN SOUTHWEST UGANDA}

doi:10.1136/archdischild-2013-303966.050

1.2 $\mathrm{K}$ MacLeod, ${ }^{3} \mathrm{~N}$ Sandercombe, ${ }^{3} \mathrm{~A}$ Barrett, ${ }^{3} \mathrm{E}$ Heffernan, ${ }^{2 \mathrm{H}}$ Kalema, ${ }^{2} \mathrm{~J}$ Ngonzi, ${ }^{2} \mathrm{G}$ Mugyenyi. ${ }^{\prime}$ St Helens and Knowsley NHS Trust, Merseyside, UK; ${ }^{2}$ Mbarara University of Science and Technology, Mbarara, Uganda; ${ }^{3}$ Peninsula College of Medicine and Dentistry, Exeter and Plymouth, UK

Background There is an increasing incidence of low birth weight babies $(<2500 \mathrm{~g})$ in Uganda. This can cause significant perinatal morbidity and mortality and is related to intrauterine growth restriction (IUGR). Estimated fetal weight (EFW) can be established clinically or sonographically and influences obstetric management. The accuracy of clinical and sonographic formulae needs to be established in Uganda due to resource limitations and increasing burden of IUGR.

Methods We prospectively studied term, cephalic, singleton pregnancies in Mbarara Regional Referral Hospital, Uganda. Clinical EFW was calculated using Dare's and Johnson's formulae, sonographic EFW used Hadlock C formula. Effects of the following variables were also examined: maternal age, body mass index, parity, gestational age, fetal sex and birth weight.

Results Ninety women were enrolled. Birth weight was correctly estimated $( \pm 10 \%)$ in $25.6 \%, 47.8 \%$ and $64.4 \%$ of the cases using Johnson's, Dare's and Hadlock's formulae respectively. Accuracy levels differed between the three formulae $(p<0.001)$. Johnon's is significantly less accurate than other formulae, whilst Hadlock's is significantly more accurate. Nine percent of deliveries had birth weight $<2500 \mathrm{~g}, 88 \%$ had birth weight of $2500-4000 \mathrm{~g}$, while $3 \%$ weighed $>4000 \mathrm{~g}$. The sensitivity of predicting birth weight of less than $2500 \mathrm{~g}$ was only $50 \%$ for ultrasound and $13 \%$ for clinical EFW, with $95 \%$ and $100 \%$ specificity respectively.

Conclusion Sonographic EFW is more accurate than clinical formulae. It best at predicting low birth weight fetuses, however, sensitivity remains low and better methods for identification need to be developed.

\section{PF.40 WITHDRAWN BY AUTHOR}

\section{PF.41 'TO HAVE A LITTLE BIT OF HOPE IS LIKE BEING THROWN A LIFELINE' THE EXPERIENCE OF PREGNANT WOMEN WITH A DIAGNOSIS OF FETAL LOWER URINARY TRACT OBSTRUCTION (LUTO)}

doi:10.1136/archdischild-2013-303966.051

${ }^{1,3}$ EQ-J Quinlan-Jones, ${ }^{2}$ ED Denny, ${ }^{1,3} \mathrm{MK}$ Kilby. 'University of Birmingham, Birmingham, UK; 'Birmingham City University, Birmingham, UK; ${ }^{3}$ Fetal Medicine Department, Birmingham Women's Hospital NHS Foundation Trust, Birmingham, UK

Introduction The PLUTO: Percutaneous Shunting for Lower Urinary Tract Obstruction (LUTO) Study was a multi-centre, randomised, controlled trial (RCT) undertaken to evaluate the safety and efficacy of fetal vesicoamniotic bladder shunting in moderate to severe ante-natally diagnosed cases compared to conservative management. Within the trial a qualitative study explored women's motivation for entering the trial, and their experience of the condition and its management. The trial was terminated early, but the qualitative data collected provided an insight into being pregnant with a fetus with a serious medical condition.

Aim To gain insight into the experiences and perceptions of pregnant women asked to participate in an interventional fetal medicine trial requiring an invasive procedure.

Method Semi-structured interviews were undertaken with a purposive sample of women involved in the PLUTO trial $(n=6)$. The data were analysed thematically.

Findings Motivation for participation in the PLUTO trial was consistent with other research, and involved reasons of both altruism and self-interest. Loss of a normal pregnancy was precipitated by the current routine use of ante-natal scanning. This was associated with uncertainty for women and a decision making process that could only result in a less than ideal option.

Conclusion Undertaking a qualitative study within the PLUTO trial has illuminated the experience of receiving a prenatal diagnosis of LUTO following ultrasound scanning. The unexpected nature of the diagnosis itself and the evident uncertainty that permeates this condition and its management during pregnancy appears to exert some influence over women's decision making.

\section{PF.42 UNFUSED AMINION AND CHORION (UAC) AFTER 15 WEEKS GESTATION AND ASSOCIATION WITH CHROMOSOMAL ABNORMALITIES}

doi:10.1136/archdischild-2013-303966.052

'S Raouf, 'C Adcock, 'D Hay, ${ }^{2} \mathrm{~J}$ Ashworth. 'Royal Derby Hospitals NHS foundation trust, Derby, UK; ${ }^{2}$ Univercity of Nottinghan, Nottingham, UK

Objectives To assess a possible association between UAC and Trisomy 21.

Method Medical records of all women undergoing amniocentesis between April 2008 and October 2012 at RDH were analysed highlighting UAC and karyotype result.

Results 425 women underwent amniocentesis for standard screening rationale.

33 fetuses were diagnosed with trisomy 21, (incidence of 7.6\%).

21 women had their amniocentesis delayed because of UAC (404 did not as the membranes were normally fused - NFM). Of these, 10 had a result confirming the presence of Trisomy 21, the remaining 11 had a normal result, 23 of the 404 with NFM had Trisomy 21. 


\section{Abstract PF.42 Table}

\begin{tabular}{lllc}
\hline & Down's present & Down's absent & Total \\
\hline UAC & 10 & 11 & 21 \\
NFM & 23 & 381 & 404 \\
& 33 & 392 & 425 \\
\hline
\end{tabular}

Sensitivity of UAC $=10 / 33=30.3 \%$

Specificity of UAC $=381 / 392=97.2 \%$

$\mathrm{PPV}=10 / 21=47.6 \%$

$\mathrm{NPV}=381 / 404=94.3 \%$

Conclusion We suggest that NFM has a negative association with Trisomy 21 with high NPV and may be helpful in counselling. Furthermore UAC seems to be only associated with Trisomy 21 and no other chromosomal abnormality in this population. We suggest further prospective study of this phenomenon. Abnormalities of cell adhesion molecules (encoded on C21) are well described in Down's (DSCAM - Down's Cell Adhesion Molecule) and this suggests a possible aetiology.

\section{PF.43 LOW MATERNAL SERUM PAPP-A IN THE FIRST TRIMESTER AND PREGNANCY OUTCOME: AN EXPERIENCE OVER 3 YEARS}

doi:10.1136/archdischild-2013-303966.053

A Weaver, S Nanda, C Rozette, P Kyle, S Sankaran. Women's Health, St Thomas' Hospital, London, UK

Introduction Maternal serum PAPP-A (pregnancy associated plasma protein-A) is a part of combined screening. Previous studies have shown correlation between low PAPP-A and adverse pregnancy outcome.

Objective The aim of this study is to establish the positive predictive value of low-PAPP-A in prediction of adverse pregnancy outcomes - pre-eclampsia (PET), pregnancy induced hypertension (PIH), delivery of small for gestational age neonates (SGA) and late pregnancy losses.

Materials and Methods 16690 women underwent combined screening from $1 / 8 / 2008$ to $1 / 8 / 2011$. 326 women with low PAPP-A $(=<0.3 \mathrm{MoM})$ were identified $(1.95 \%)$. The median PAPP-A of the screening population was $1.074 \mathrm{MoM}$. Within this group of pregnancy with low PAPP-A, maternal serum PAPP-A was compared between the subgroups of adverse pregnancy outcome and normaloutcome.

Results

\section{Abstract PF.43 Table}

\begin{tabular}{lrlll}
\hline Cases & N & $\begin{array}{l}\text { PAPP-A MoM } \\
\text { (Median) }\end{array}$ & p & PPV \% \\
\hline Normal outcome & 146 & 0.255 & - & - \\
Adverse outcome & 95 & 0.225 & $0.004^{*}$ & 39.4 \\
PET & 25 & 0.234 & 0.184 & 10.8 \\
PIH & 10 & 0.243 & 0.769 & 4.1 \\
SGA & 44 & 0.220 & $0.004^{*}$ & 21.4 \\
Late pregnancy losses & 15 & 0.191 & $0.024^{*}$ & 6.6 \\
\hline * Adjusted
\end{tabular}

* Adjusted significance level $\mathrm{P}<0.0125$ - post hoc Bonferroni correction

Conclusion In our screening population, median PAPP-A MoM was higher compared to some previous studies. Maternal serum PAPP-A in pregnancies with adverse outcome was significantly lower than those that resulted in a normal outcome. Compared to the pregnancies with low-PAPP-A but normal outcome, median PAPP-A MoM was significantly lower in pregnancies ending in delivery of small-for-gestational age neonate (customised BW $<10^{\text {th }}$-centile), and showed a trend towards a decrease in those ending in late-pregnancy losses (>24 weeks)

\section{PF.44 WITHDRAWN BY AUTHOR}

\section{PF.45 COMPARISON OF ANTENATAL DETECTION RATES OF FETAL ANOMALIES BETWEENEIGHT TRUSTS IN THE EAST MIDLANDS AND SOUTH YORKSHIRE. ARE THE FETAL ANOMALY SCREENING PROGRAMME TARGETS ACHIEVABLE?}

doi:10.1136/archdischild-2013-303966.054

'MW Osman, ' JLS Budd, ' ${ }^{2}$ East Midlands \& South Yorkshire Congenital Anomaly Register, Dept. Health Sciences, University of Leicester,, Leicester, UK

Background In 2010, the UK Fetal Anomaly Screening Programme (FASP)introduced targets varying between $50 \%$ and $98 \%$ for the antenatal detectionof elevencongenital anomalies*. Auditing these standards is complex, requiring an understanding oftrust size, case mix, the rarity of anomalies, screening uptake and the local obstetric population.

Aim To compare antenatal detection rates achieved by eight trusts within the region covered by the East Midlands and South Yorkshire Congenital Anomaly Register (EMSYCAR) between 2010 and 2011.

Methods Data were obtained for 651 cases, identified by relevant ICD-10 codes, delivered between $1 / 1 / 10$ and 31/12/11. Bilateral renal agenesis and 'lethal skeletal dysplasias' were excluded, as the number of reported cases was too smallto be reliable. The EUROCAT* definition of thirteen 'serious' cardiac conditions was adopted. For those cases detected antenatally, gestational age at diagnosis was recorded, and the booking hospital anonymised. Eight trusts of varying size were analysed.

Results Although the vast majority of FASP cases were identified before delivery, only the anencephaly target was met by all eight, while Spina Bifida and Trisomy 18 targets were missed by five. One trust reached only four of nine targets, missing three of the others by a single case.However, none of the FASP targets was achieved by $20+6$ weeks.

Conclusions Most trusts met expected antenatal detection rates specified by FASP, but not by $<20+6$ weeks. Considerable variability exists both between trusts and anomalies. Data produced here should enable the precise training needs of each trust to be identified more accurately.

\section{PF.46 CHILDHOOD HOSPITAL ADMISSIONS OF CHILDREN CONCEIVED FOLLOWING ASSISTED REPRODUCTIVE TECHNOLOGY}

doi:10.1136/archdischild-2013-303966.055

MH McComiskey, C Patterson, M Stevenson, IE Cooke. Queen's University Belfast, Belfast, UK

The purpose of this project was to compare paediatric hospital admission rates of children conceived via assisted reproductive technology with that of the population as a whole.

Consent-based ART register and admission records were linked and comparisons made between admission rates in the general population and the ART cohort by calculation of standardised admission ratios (SAR's). The project was performed in accordance with HFEA regulations and had ethical approval.

Children conceived via ART had a significantly lower rate of hospital admissions (all admissions and first hospital admissions) than 